\section{Influence of Illumination Conditions on Temperature in Sample Cell and the Output of a Quadrant Detector in an Optical Tweezers System}

Yuqiang Jiang, ${ }^{1,2,3}$ Honglian Guo, ${ }^{2}$ Chunxiang Liu, ${ }^{2}$ Zhaolin Li, ${ }^{2}$ Bingying Cheng, ${ }^{2}$ Daozhong Zhang, ${ }^{2}$ and Suotang Jia ${ }^{1}$

${ }^{1}$ College of Physics and Electronics Engineering, Shanxi University, Taiyuan, P. R. China

${ }^{2}$ Institute of Physics and Center for Condensed Matter Physics, Chinese Academy of Science, Beijing, P. R. China

${ }^{3}$ Shanxi Datong University, Datong, P. R. China jyq@cybox.co.jp

\section{Introduction}

In an optical tweezers system, the output signal of a photodiode quadrant detector and the temperature in a sample cell are two key factors for the quantitative measurements of mechanical properties of living biological objects such as cells, organelles and macro-molecules. In order to enhance the output of a quadrant detector and effectively control the temperature in a sample cell, the dependence of the temperature in the sample cell and the output of the quadrant detector for different illumination conditions are studied. The results show that appropriate illumination conditions can ensure both nearly constant temperatures in the cell and the desired output signal, which provides for the possibility of highprecision and damage-free analysis of living biological objects.

In an optical tweezers system, a CCD camera and a photodiode quadrant detector (QD) are employed to make displacement measurements. The precision of a CCD measurement is superior, so CCD measurements are routinely used in experiments of tiny displacement measurements, such as the measurement of the 8-nm step displacement of a kinesin motor. ${ }^{[1]}$ However, the time response of a CCD is very limited, so for measurements of rapid motion, such

Fig. I Thermocouple Calbration. The thermocouple is put into a water bath at different temperatures. The temperature of the water is measured by a calibrated thermometer to $\pm 0.1^{\circ} \mathrm{C}$. The thermocouple output microvoltmeter data is fitted with a quadratic function. The standard deviation of this fit is $0.133^{\circ} \mathrm{C}$, yielding a thermocouple temperature measurement accuracy of $0.5^{\circ} \mathrm{C}$.
Fig.2 Schematic diagram of path illumination path for the optical tweezers. Infrared light is blocked by the heat filter. Symmetrical illumination light is obtained with a ground-glass diffuser. Kohler illumination is formed with the Field diaphragm, aperture diaphragm, and condenser (C1, C2). Piezo-electric-transducers (PZT) are used for microoperation of the sample cell and force calibration; The sample in the object plane of the objective is imaged on the surface of the $Q D$.

as the measurement of the step of myosin motor ${ }^{[2]}$ a QD is usually used instead of a CCD.

The sensitive wavelength range of most QDs is in the near infrared, which happens to be the region of strong heat absorption of water. In order to avoid water absorption-induced temperature increases in the sample cell, the infrared wavelength components of the microscope standard illumination are blocked. This has little effect on displacement measurements with a CCD. However, this will result in a decreased output signal from a QD, which, at best, is only in the range of tens of millivolts, while the noise signal from a QD is in the level of millivolts-making the signal-to-noise ratio (SNR) of the measurement system problematical. To increase the SNR, the standard illumination conditions of the microscope should be changed to guarantee the required output signal of the QD, but the consequence of this change is to maximize the near infrared light. This will bring about a temperature rise in sample cell because of water absorption, which is usually fatal for the study of living objects.

The volume of the sample cell in an optical tweezers is usually very small-only about $100 \mu \mathrm{l}-\mathrm{so}$ the temperature in a sample cell is easily affected by its environment. The effect of an optical laser on the temperature in a sample cell has been studied in the references ${ }^{[3-5]}$ and laser-induced cell damage with different wavelengths has been reported.$^{[6-9]}$ Using an infrared trap laser as the input of a QD can greatly increase the output of the QD while having little influence on the temperature inside the sample cell. ${ }^{[5,10,11]}$ In this way, however, the relationship of the motion of a bead image on the QD plane and the actual motion of a bead in the objective plane is nonlinear, and this will make it very difficult to exactly calibrate the output of a QD and the trap stiffness. Certainly it can be considered as linear within a certain range of deviation from the trap center, but the scope is very limited when a trap laser is 


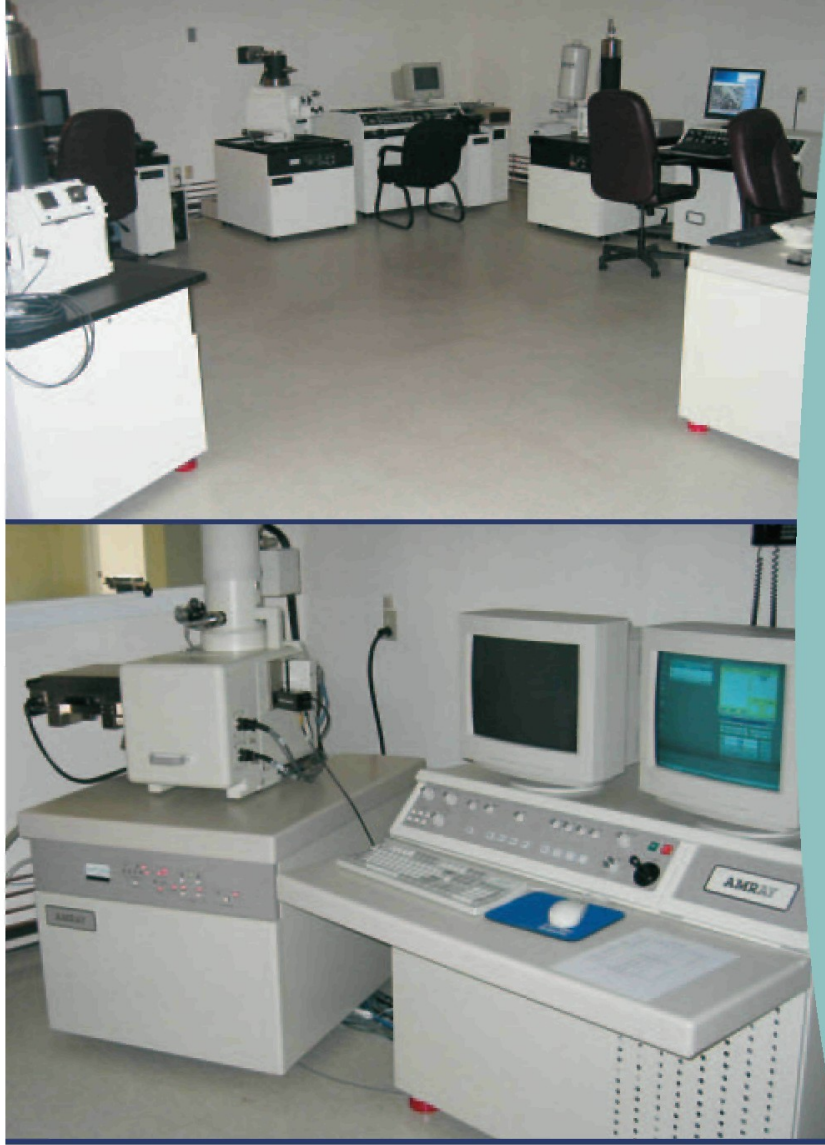

\section{Pre-Owned SEMs -}

\section{The Smart \& \\ Easy Alternative \\ to Buying New}

Why buy a new Scanning Electron Microscope when SEMTech Solutions offers a smarter and easier solution?

We specialize in pre-owned, rebuilt and upgraded products, including:

- Field Emission SEMs

- Digital Imaging

- Computer and EDS Upgrades

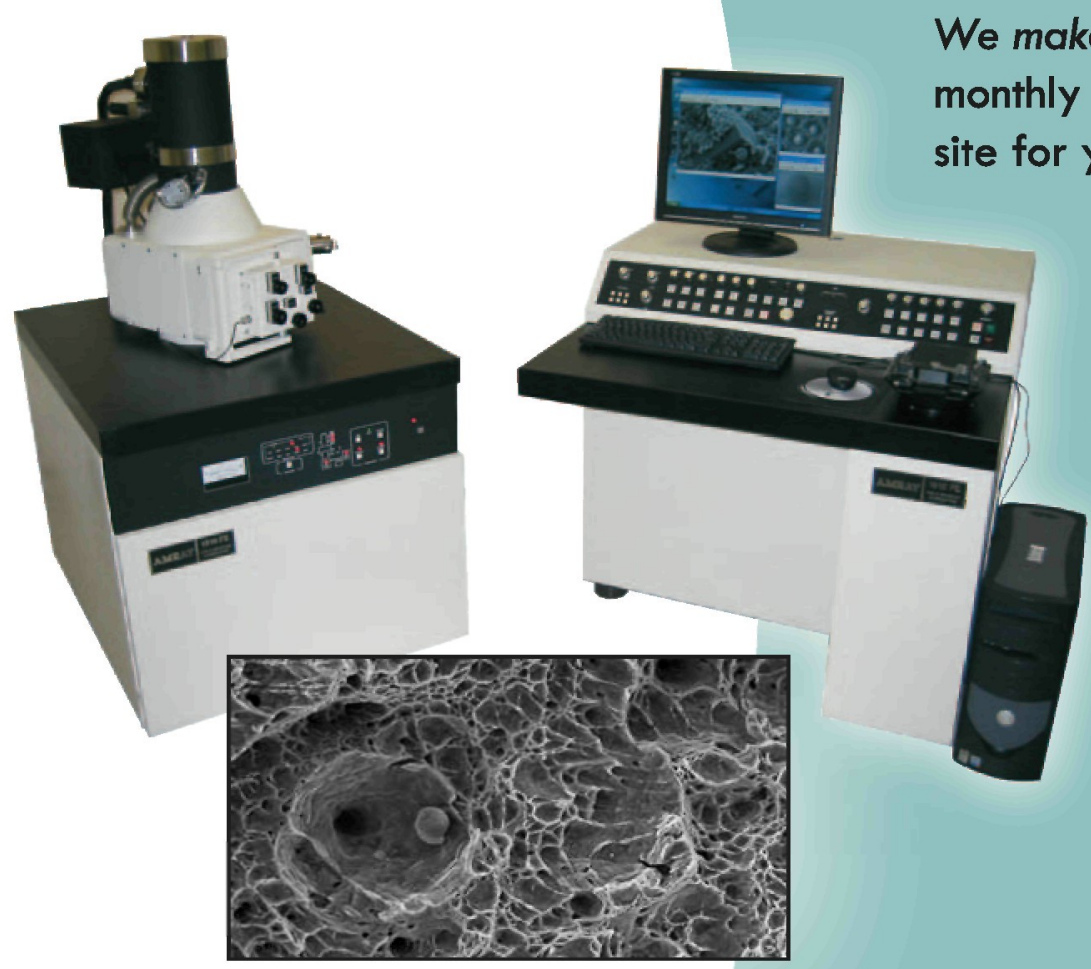

We make it easy by offering hands-on, in-person equipment demonstrations at our Massachusetts facility. Scheduling a demo is simple; just call us at

$978 \cdot 663 \cdot 9822$ for an appointment.

Visit us at

www.semtechsolutions.com to get more information on all SEMTech Solutions products and services.

Smart. Easy.

- SEMTech Solutions, Inc.

VEMTech Solutions, Inc.

6 Executive Park Drive

North Billerica, MA 01862

$978 \cdot 663 \cdot 9822$

www.semtechsolutions.com 
Fig. 3 Dependence of the temperature in the sample cell and the output of the $Q D$ on the voltage of illumination lamp. (a) With the heat filter in the illumination light path. (b) Without the heat filter in the illumination light path. The voltage of the lamp is changed from $2.5 \mathrm{~V}$ to $12 \mathrm{~V}$. The temperature in the sample cell is monitored with a thermocouple system. At each point, when the temperature is stable (illumination time duration is about 2 mins), the value of temperature in the sample cell and the output of the $Q D$ are recorded. used as an input of a QD.

An ideal arrangement would be to use an infrared laser as an input to the QD instead of the standard illumination light of the microscope in the illumination path. But, this needs a larger laser and considerable changes to the microscope structure.

Fig. 4 Dependence of the temperature in the sample cell and the output of the $\mathrm{QD}$ on the field diaphragm aperture size.
Fig.5 Dependence of the temperature in the sample cell and the output of the $Q D$ on the voltage of light lamp when the aperture of the field diaphragm is set to correspond to the field-of-view.

Comparatively, using the illumination light of the microscope as the input to a QD is a convenient method if it is feasible to compensate for the heating effects of the infrared component of the illumination light. Few reports, however, concern the influence of illumination light on the temperature and output signal of QD.

In order to find the desired illumination conditions for both constant temperature and a larger output signal from the QD, we designed a special sample cell, which is equipped with a thermocouple and a cooling sub-system. The influence of different illumination conditions on the temperature in the sample cell and output signal of the experimental QD is studied systematically.

\section{Materials and Methods}

The optical tweezers system consists mainly of an inverted optical microscope ((Leica DMIRB, Germany) with a high numerical aperture objective (HCXAPO $100 \times /$ NA 1.30) and a photodiode quadrant detector (Hamamtsu1557-03). The sample cell with a built-in temperature sensor is designed by ourselves and its volume is about $150 \mu \mathrm{l}(\phi 10 \times 2 \mathrm{~mm})$. The sensor is a thermocouple made of copper and constantan, and its position in the sample cell is adjustable so that the temperature in different locations in the sample cell can be measured. The temperature calibration curve is shown as Fig.1, the accuracy of the temperature measurement is $\pm 0.5^{\circ} \mathrm{C}$. The schematic diagram of the optical tweezers system can be seen in reference [12], the only difference is that the CCD camera is replaced by the QD. The detailed illumination light path is shown in Fig.2.

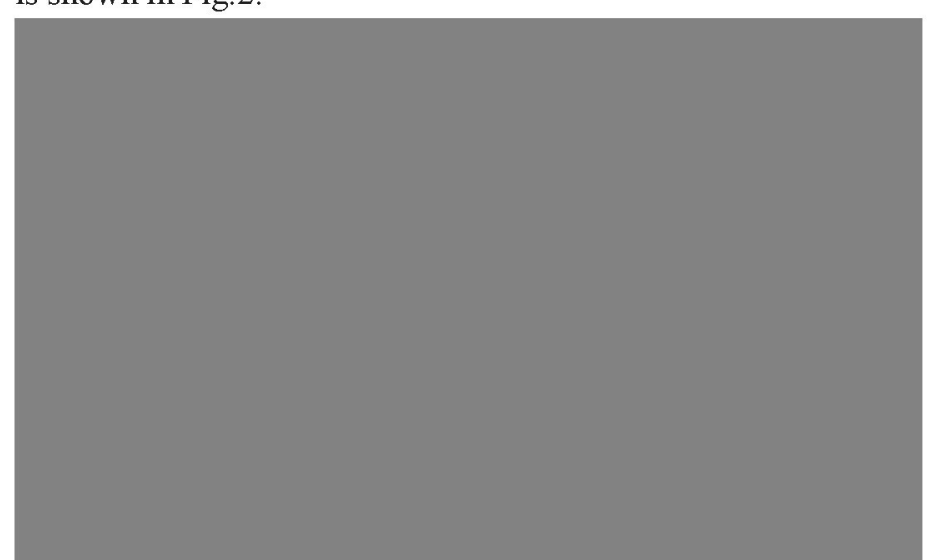

Fig. 6 Dependence of the temperature in the sample cell and the output of the $\mathrm{QD}$ on the value of the aperture diaphragm. 


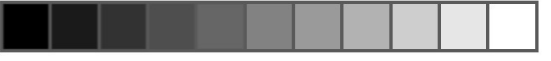

D I G I T A L C A M E R A S F O R T E M M I C R O S C O P Y

\section{The Perfect Winning Hand - TEM Camera Solutions \\ by Soft Imaging System}

The stakes are high. The competition tough.

Deal yourself the best available hand in the TEM

sector. Soft Imaging System's extensive range

of TEM hardware and software solutions places

the perfect set of tools at your fingertips. Meet

steadily rising demands in the transmission electron

microscopy business. Whether your application

is in the biomedical or materials analysis sector.

Our product range includes high-resolution CCD

cameras for wide-angle ports and bottom-mounted

positions on all standard TEMs. The cameras are

fiber or lens coupled, offer up to 11 MegaPixels

and provide dynamic ranges of up to 16 bits.

All cameras are cooled and highly sensitive with

fast readout rates, and use advanced techniques

to ensure optimal signal-to-noise ratio. The cameras

are easy to install, generally without any major

changes to your microscope. All cameras are

integrated with our TEM imaging software platform,

iTEM.

www.soft-imaging.net/tem

\section{TEM Cameras}

Cantega2k, Morada,

MegaView III, KeenView

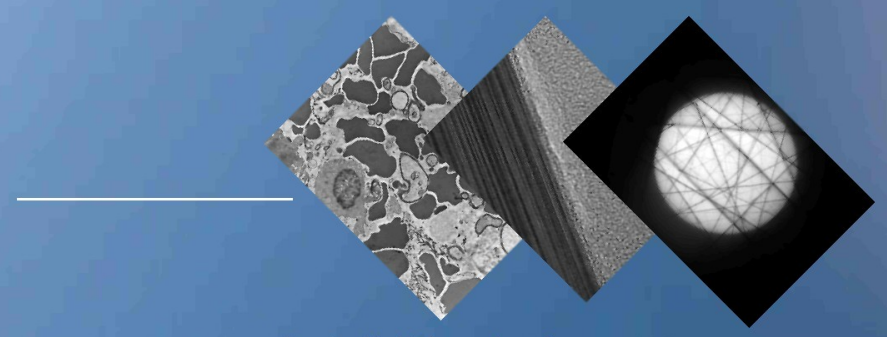

For detalled information please contact:

Soft Imaging System

info.de@soft-imaging.net

www.soft-imaging.nettem

North America: (888) FIND SIS + 1 (303) 234-9270

Europe: +49 (251) 79800-0

Asia | Pacific: +60 (3) $8313-1400$ 
Firstly, we shall study the influence of the heat filter in the illumination path and the voltage of the illuminating lamp on the temperature inside the sample cell and the output signal of the QD. Under standard illumination conditions of the microscope, i.e. with the heat filter in place, the dependence of the temperature and output signal on the voltage of the illumination lamp is measured under the condition of maximum field and aperture diaphragms openings. The output signal of the QD is determined as follows: first, a 1- $\mu \mathrm{m}$-diameter polystyrene bead is trapped by the laser tweezers, then we adjust the position of the QD to make sure that the bead image is in the center of the QD. At this time, the output of the QD should be zero, we then adjust the QD to make the QD center deviate from the trap center until the output of QD reaches its maximum. In our experiment, the QD is moved $0.3 \mathrm{~mm}$ from its center position, which is corresponding to $500 \mathrm{~nm}$ displacement in the object plane of objective. By comparing the maximum outputs, the influence of illumination conditions on the output of the QD can be determined. Fig. 3a shows the dependence of temperature and output signal on the voltage of the lamp at room temperature $\left(20^{\circ} \mathrm{C}\right)$. It can be seen that the temperature in the sample cell changes from $26^{\circ} \mathrm{C}$ to $33^{\circ} \mathrm{C}$ with an increase of voltage from $2.5 \mathrm{~V}$ to $12 \mathrm{~V}$. Despite the fact that the temperature change is not very large, the output signal of QD is small. When the voltage reaches its maximum of $12 \mathrm{~V}$, the output of the $\mathrm{QD}$ is only $55 \mathrm{mV}$. Allowing for its linear range, the output signal that can be best used in practice is about $30 \mathrm{mV}$. Under this condition, the nanometer steps of some biological motor proteins corresponds to just a few millivolts output signal from the QD. Such a small signal is obviously not enough for the quantitative measurements of small displacements.

In order to enhance the SNR of the QD, an improved design for the illumination light path is put forward. We take away the heat filter from the light path. Then the light sensitivity of the $\mathrm{QD}$ is compensated for. Fig. $3 \mathrm{~b}$ is the dependence of temperature and output signal on the voltage of the lamp; the illumination conditions are similar to that in Fig.3a except that the heat filter is removed in this experiment. From Fig.3b we can see that the output of the QD increases almost linearly from $28 \mathrm{mV}$ to 450 $\mathrm{mV}$ with the increase of lamp voltage. This results show that the output of the QD is an order of amplitude larger than that with the heat filter in the illumination path, increasing the SNR of the QD greatly. Taking this advantage into consideration, it can be concluded that the heat filter should be taken away. However, its unfavorable aspect is that the temperature in the sample cell increases greatly without the heat filter in place. When the lamp voltage is set to $12 \mathrm{~V}$, the temperature in the sample cell can reach $80^{\circ} \mathrm{C}$, which is fatal for living biological samples.

To guarantee both a large output signal from the QD and a minimal temperature increase in the sample cell, the effect of different illumination conditions, such as varying the apertures of the field diaphragm and on the output of the QD, while monitoring the temperature in the sample cell are studied after removing the heat filter from the light path. The changes of the output of the QD and the temperature in the sample cell with the aperture of the field diaphragm are shown in Fig.4, where the lamp voltage is set to be $12 \mathrm{~V}$ and the aperture diaphragm opened to its maximum. Similar results are obtained with different lamp voltage and the aperture diaphragm. So we take the Fig. 4 case as an example to analyze. It can be seen from Fig. 4 that as long as the aperture of the field diaphragm is not less than the field of view $(1.4 \mathrm{~mm}$ diameter of diaphragm or $200 \mu \mathrm{m}$ in the object plane of the objective), there is no obvious effect on the output of the QD, whereas the temperature in the sample cell is affected strongly by opening or closing the field diaphragm. The temperature varies from $80^{\circ} \mathrm{C}$ to $23^{\circ} \mathrm{C}$ as the field diaphragm closes from its maximum $(\phi 30 \mathrm{~mm})$ to minimum $(\phi 0.5 \mathrm{~mm})$, which indicates that when the aperture of field diaphragm is adjusted to a suitable size, both the large output of the QD and the small temperature increase in the sample cell can be assured. Fig. 5 is the dependence of the QD output and the temperature in the sample cell while varying the lamp voltage under the condition that the field diaphragm is set to correspond to the field of view and the aperture diaphragm opened to its maximum. It shows that there is no obvious temperature change with increase in lamp voltage while the output of the QD increases greatly.

Next, the effect of varying the aperture diaphragm on the temperature and the output signal is studied. In this experiment, the field diaphragm is fixed to correspond to the field of view and the lamp voltage is set to $12 \mathrm{~V}$. Fig. 6 shows the change of temperature in the sample cell and the output of the QD with different aperture diaphragm sizes. It can be seen that the output signal of the QD changes greatly with adjusting the aperture diaphragm while the effect on the temperature is small. With opening of the aperture diaphragm, the output of the QD increases at first, then reaches a maximum when the aperture of the diaphragm is 8 , and then decreases slowly.

\section{Discussion}

For optical tweezers combined with a QD as a means to measure the displacement and force of moving micro objects, the ideal illumination condition requires both a high SNR and a constant temperature in the sample cell.

The SNR of QD can be described as follow:

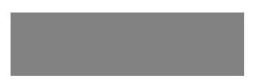

Where $V_{N}$ is noise voltage of the QD. It can be determined by measuring the output of a QD when a fixed bead in the object plane of the objective is imaged on the center of the QD. For a stable system, $V_{N}$ can be considered to be a constant. $V_{s}$ is the signal output of the QD corresponding to the trapped bead motion. This is related directly to the displacement of the bead and the intensity of the effective illumination light. $V_{s}$ can be expressed as:

Where $S$ is the sensitivity of the photodiode, and $k$ is a constant that is related to the magnification factor of the electric circuit of the QD. $I_{m}$ is the light intensity of the bead image; $I_{n}$ is the light intensity of the background, $\Delta x$ is the displacement of the bead image on the QD, and $r$ is the radius of the bead image on the QD.

For biological experiments, such as myosin and kinesin, the displacement step is usually very small. So, to acquire a desired high $V$, the intensity of the illumination light that is sensitive to the QD has to be enhanced. However, the sensitive wavelength 
range of most QDs is within the near infrared, which will inevitably raise the temperature in the sample cell.

Taking all these situations into account, the conclusion can be drawn from our experimental results that in order to obtain the larger output signal and minimal temperature change, the heat filter should be removed from the light path and the field diaphragm and aperture diaphragm should be adjusted to appropriate sizes.

For the situations shown in Fig. 5 and Fig.6, when the heat filter is removed, the lamp voltage is set to $12 \mathrm{~V}$, the field diaphragm corresponds to the view field and the aperture diaphragm is in position 8, the influence of the illumination conditions on the SNR compared with standard illumination shown as Fig. 3 can be estimated. After removing heat filter from the illumination path, the output of the QD increases from $55 \mathrm{mV}$ to $450 \mathrm{mV}$ while the temperature changes no more than $3^{\circ} \mathrm{C}$. The change of $V_{N}$ can be neglected, but the SNR can be improved:

$$
\begin{aligned}
& \Delta_{S N R}=\Delta V_{S} / V_{N}=(450-55) / V_{N} \\
& \delta_{S N R}=\Delta_{S N R} / S N R \cong 800
\end{aligned}
$$

This is a very favorable result for both the signal strength from the QD and for maintaining a constant temperature in the sample cell, providing for the possibility of quantitative measurements of nanometer displacement and piconewton forces.

\section{References:}

1. A. D. Mehta, M. Reif, J. A. Spudich, D. A. Smith and R. M. Simmons, "SingleMolecule Biomechanics with Optical Methods," Science 283, 1689-1695 (1999).

2. J. T. Finer, R. M. Simmons, and J. A. Spudich, "Single Myosin Molecule Mechanics: Piconewton Forces and Nanometer Steps," Nature 368, 113-119 (1994).

3. W. W. LeCates, S. C. Kuo and W. E. Brownell, Association for Research in Otolaryngology Midwinter Meeting. Abstract 622 (1995).

4. Y. Lui, D. K. Cheng, G. J. Soneck, M. W. Berns, C. F. Chapman, and B. J. Tromberg, "Evidence for Localized Cell Heating Induced by Infrared Optical Tweezers," Biophys. J. 68, 2137-2144 (1995).

5. Y. Lui, D. K. Cheng, G. J. Soneck, M. W. Berns and B. J. Tromberg, "Microfluorometric Technique for the Determination of Localized Heating in Organic Particles," Appl. Phys. Lett. 65 919-921 (1994).

6. D. Mietchen, T. Schnelle, T. Müller, Hagedorn and G. J. Fuhr, "Automated Dielectric Single Cell Spectroscopy - Temperature Dependence of Electrorotation," J. Phys. D: Appl. Phys. 35 1258-1270 (2002).

7. A. Ashkin, J. M. Dziedzic, T. Yamane, "Optical Trapping and Manipulation of Single Cells Using Infrared Laser Beams," Nature 330 769-771(1987).

8. A. Ashkin, K. Schutze, J. M. Dziedzic, U. Euteneuer and M. Schliwa, "Force Generation of Organelle Transport In Vivo Measured by an Infrared Laser Trap," Nature 348 346-348 (1990)

9. M. W. Berns, J. R. Aist, W. H. Wright and H. Liang, "Optical Trapping in Animal and Fungal Cells Using a Tunable, Near-infrared Titanium-sapphire Laser," Exp. Cell Res. $198375-378$ (1990).

10. G. V. Shivashankar, G. Stolovitzky and A. Libchaber, "Backscattering From a Tethered Bead as a Probe of DNA Flexibility," Appl. Phys. Lett., 73, 291-293 (1998).

11. L. P. Ghislain, N. A. Switz and W. W. Webb, "Measurement of Small Forees Using on Optical Trap," Rev. sci. instrum. 65 2762-2768 (1994)

12. H. L. Guo, X. C. Yao, Z. L. Li, B. Y. Cheng, X. H. Han and D. Z. Zhang, Sci. in Chin. 45919 (2002).

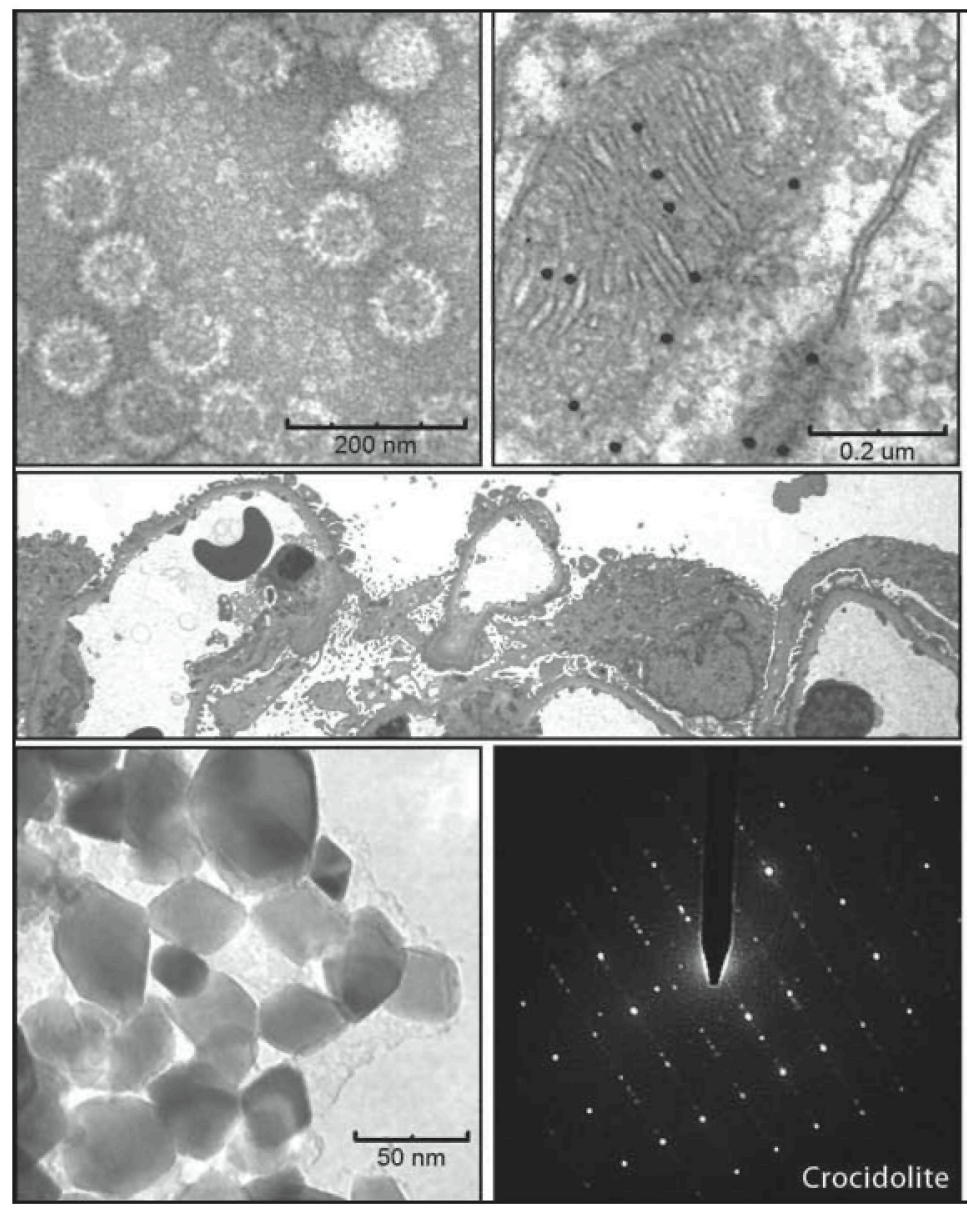

\section{Affordable TEM} camera systems for research, healthcare,

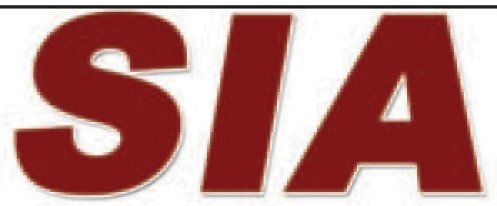
education, and industry since 2001

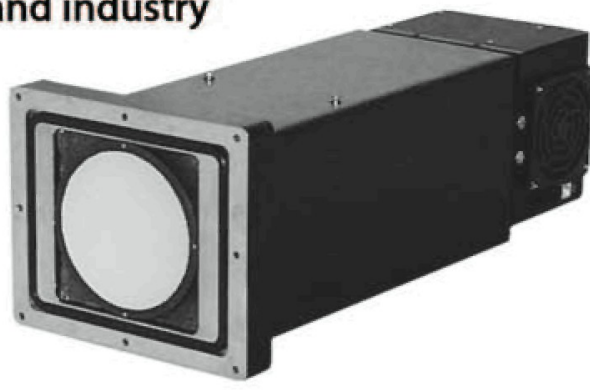

1 to 16 Megapixels, slow scan and TV

Magnification factor of 1 on bottom mounted cameras Diffraction beam stop on side mounted cameras

Reliable, easy to use and upgrade

\section{Standard and custom configurations for any TEM}

Compatible with existing TEM accessories

Scientific Instruments and Applications

2773 Heath Lane; Duluth, GA; 30096

(770) 232 7785; www.sia-cam.com

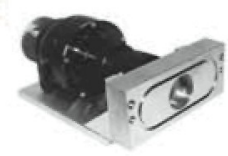

